\title{
Perceived barriers to access available HIV and sexually transmitted infection services among men who have sex with men (MSM) in Tanga Region, Northern Tanzania
}

\author{
Hamimu O. Kigumi ${ }^{1,2}$, Sia E. Msuya², Damian J. Damian ${ }^{2}$ \\ ${ }^{1}$ Local Government Training Instituite, Tanzania \\ ${ }^{2}$ KI\Kilimanjaro Christian Medical University College, Tanzania
}

\begin{abstract}
Introduction: Studies have shown high prevalence of human immunodeficiency virus (HIV)/sexually transmitted infections (STIs) among men who have sex with men (MSM) globally. Other studies have shown that barriers to accessing the HIV/STI services from the health facilities are among the factors that contribute to the increased prevalence among MSM. This study was aimed at determining the proportion of MSM who accessed health care and disclosed their sexual orientations to health workers (HWs) and anticipated barriers if they disclosed their sexual orientation.

Material and methods: A cross sectional study with both quantitative and qualitative methods was conducted from April to June 2015 in four districts of Tanga, Tanzania. Two hundred and sixty-six MSM were enrolled in the study using the respondent-driven sampling method. Quantitative data were collected using a structured administered interview and entered in SPSS 23.0 for analysis while qualitative data were collected using in-depth interview and focus group discussion, analyzed and interpreted.

Results: The mean age of the participants was 27.2 (SD \pm 6.7 ) years, $48 \%$ were married or cohabiting. $68.8 \%$ of MSM had not accessed HIV/STI services until they were sick. $13.4 \%$ never attended the health facilities for treatments even if they were sick due to fear of stigma and discrimination. $67.8 \%$ had ever disclosed their sexual orientation to HWs.

Conclusions: MSM need to be empowered to overcome their perceived fears towards health care workers. Efforts should be put into breaking the cycle of negative information and perceptions of MSM from HWs and the community.
\end{abstract}

HIV AIDS Rev 2019; 18, 2: 115-119 DOI: https://doi.org/10.5114/hivar.2019.85923

Key words: HIV, barriers, MSM, Tanzania, STIs.

\section{Introduction}

The term "men who have sex with men" (MSM) is an inclusive public health term encompassing both gay men and bisexual men who engage in sexual activity with other males,

regardless of their motivation for doing so or identification of a specific sexual orientation $[1,2]$.

The Joint United Nations Programme on HIV and AIDS (UNAIDS) reports that human immunodeficiency virus (HIV) continues to be a major public health concern

Article history:

Received: 12.12.2018

Received in revised form: 31.12 .2018

Accepted: 05.02.2019

Available online: 20.05.2019
International Journal of HIV-Related Problems

HIV \& AIDS

R e vi e w 
especially in sub-Saharan Africa (SSA). A combination of multiple behavioral and medical interventions including antiretroviral treatment for HIV has led to a decline of new HIV infections amongst adults and children in SSA. For MSM the reverse is true about HIV trends, which increase daily $[3,4]$. MSM are a high risk population and both the incidence and prevalence of HIV in this population are increasing in both high and low-too-middle income countries $[4,5]$.

In Kenya, high HIV prevalence of $43 \%$ and incidence of 35.2 per 100 people per year for men who have sex with men exclusively has been reported [6, 7]. In Uganda studies report $42.2 \%$ prevalence of HIV in MSM compared to $5.3 \%$ in the general population and in Tanzania $22.3 \%$ HIV prevalence amongst MSM compared to $5.1 \%$ in the general population $[8,9]$.

Criminalization, stigma, discrimination of MSM, lack of national strategies for prevention and access to care for MSM in most SSA countries including Tanzania are explained as among the major factors contributing to the prevalence and incidence of HIV among MSM $[10,11]$.

MSM face various challenges when seeking healthcare services including stigma, discrimination, lack of confidentiality among healthcare workers, criminalization of homosexuality, the lack of awareness and sensitivity healthcare workers possess towards the needs of MSM [12]. The healthcare environment in most sub-Saharan countries is very unfriendly towards the MSM population $[13,14]$. The negative perception that health workers have towards MSM discourages the HIV key population to seek healthcare services such as HIV and sexually transmitted infections (STIs), preventive treatment, care and support when they are sick or not [15]. When MSM expose their status to health workers during seeking for HIV and STIs health services, health workers blame them for being MSM and some of them totally refuse to give treatment [16]. This leads to delay or avoidance of treatment for HIV and other STIs within the MSM population which results in increased prevalence $[12,17]$.

In Dar Es Salaam, Tanzania the majority of the MSM accessed HIV and STI services when they were sick and never disclosed their sexual orientation to health workers due to lack of confidentiality, fear of stigma and discrimination, shame and mistreatment at the health facilities and fear of the healthcare worker's reaction after they disclosed their sexual orientation [18].

There is limited information on accessibility of HIV and STI services among MSM in Tanzania. Most of the country guidelines for provision of HIV and STI services are directed towards other HIV key populations and heterosexual individuals while ignoring MSM. Understanding the perceptions that MSM have towards healthcare services would help providers to understand the barriers that exist among MSM and provide a basis for developing user-friendly health services.

This study was conducted in Tanga region, in NorthEastern Tanzania, to determine the local situation with regards to MSM accessing health services and providing baseline information to policy makers and HIV and STI implementers.

\section{Material and methods}

\section{Study area}

The cross-sectional study was conducted from April to June 2015 among MSM from four districts out of the eight Districts of Tanga Region, namely Tanga Municipal, Muheza, Pangani and Korogwe. Tanga region is a cosmopolitan region with a population of 2,045,205 [16] with differing origins, backgrounds, and behavior patterns that increase interactions including sexual interactions such as homosexuality. The HIV prevalence within the MSM population in the city is approximately $11 \%$ (Tanzania HIV/AIDS and Malaria Indicator Survey - THMIS, 2012). The study was based at Tanga Aids Working Group (TAWG) non-governmental organization that provides HIV and STI health services such as sexual health education, counseling, voluntary counseling and testing (VCT), care, HIV/STI prevention, treatments and referral services for the community including the most at-risk populations (MSM, sex workers, and injecting drug users) in all the eight districts of Tanga region for the past 3 years prior to the data collection.

\section{Study design and population}

A descriptive research design was used, in which both quantitative and qualitative methods were utilized. The study population recruited comprised MSM who lived in four districts of the study area, aged 18 years or older and having provided informed consent to participate. Those who refused to participate were excluded from the study.

During the study period the first five initial seeds were obtained from the MSM who attended centers to seek treatment, care or other HIV services such as condoms and VCT in TAWG centers found in each of the four districts of the study. Each participant was given three coupons to recruit the first wave of participants. Each wave then got three coupons to recruit the next wave until the sample of 266 participants was recruited. Proportional to size sampling was used to determine the number of participants to be enrolled per district. Tanga Municipality recruited more due to the higher number of MSM.

\section{Data collection}

Using a respondent-driven sampling (RDS) technique data were collected from March to June 2015. Each new recruit was then asked to recruit the same number as the initial seeds. Each subgroup is now independent which mitigates the biases that can result from a snowball technique. Prior to enrollment and signing informed consent the participants were provided with information regarding the study. Faceto-face interviews with questionnaires were conducted with 
all participants focused on gathering information on identity, barriers to access available healthcare, disclosure, community life, utilization of healthcare services, perceptions among MSM with regards to healthcare workers, and social networks used for solving social problems.

Furthermore, focus group discussion (FGD) and indepth interviews were conducted to explore in detail the perceptions of reactions of healthcare workers once MSM had disclosed their sexual orientation to them. The interviews were conducted in a private and safe environment for the participants. The interviews were developed in English but carried out in Kiswahili.

\section{Data analysis and ethical considerations}

Quantitative data were entered and analyzed using SPSS software version 23.0 whereby percentages were used to summarize categorical variables and mean or median with their respective measures of dispersion summarized continuous variables. Qualitative data were analyzed manually and interpreted.

The ethical clearance to conduct the study was received from Kilimanjaro Christian Medical University, Ethical Research Committee (CRERC). A researcher was provided with an ethical clearance number 827. Permission to conduct the study was sought from Tanga AIDS Working Group (TAWG) and from District Medical Officers (DMO) of respective districts. Only numbers were used in the questionnaire for identification of the participants to avoid disclosing personal information and protection of the participants.

\section{Results}

A total of 266 men having sex with men (MSM) were enrolled in the study. Their age ranged from 18 to 53 years; with mean $( \pm S D)$ age at enrollment being $27.2( \pm 6.7)$ years. Enrolment of study participants were Tanga Municipal 180/266 (67.7\%), 38/266 (14\%) from Muheza, 20/266 (8\%) from Pangani and 28/266 (11\%) from Korogwe districts. Most study participants had either secondary or higher education 170/266 (64\%) and were not formally employed $239 / 266$ (89.8\%). Of the 266 MSM, 212/266 (79.9\%) reported currently having partners who were men. More than half (144/266 - 54.1\%) of the participants identified themselves as bisexual and practiced both an insertive and a receptive role $127 / 266(47.7 \%)$, but the proportion of bisexuals who had female partners $(32-12.3 \%)$ differed from those who identified as homosexual (122 - 45.9\%) (Table 1).

About 182/266 (68.4\%) seek treatment for STIs to the health facility when they are serious and 38/266 (14.3\%) were self medicated by buying the drugs from the pharmacies. In seeking HIV services 185/266 (69.5\%) reported not having attended health facilities to demand either HIV voluntary counseling and testing, condoms, lubricants or HIV
Table 1. Social demographic characteristics of the participants $(n=266)$

\begin{tabular}{|c|c|c|}
\hline Factor & $n$ & $\%$ \\
\hline \multicolumn{3}{|l|}{ Place of residence } \\
\hline Tanga Municipal & 180 & 67.7 \\
\hline Muheza & 38 & 14.3 \\
\hline Pangani & 20 & 7.5 \\
\hline Korogwe & 28 & 10.5 \\
\hline
\end{tabular}$$
\text { A }
$$

Age of respondent

\begin{tabular}{l|c|c}
\hline $15-24$ & 90 & 33.8 \\
\hline $25-34$ & 134 & 50.4 \\
\hline $35-54$ & 42 & 15.8 \\
\hline
\end{tabular}

Education level

\begin{tabular}{l|c|c}
\hline None & 9 & 3.4 \\
\hline Primary & 87 & 32.7 \\
\hline Secondary and above & 170 & 63.9 \\
\hline
\end{tabular}

Employment status

\begin{tabular}{l|c|c}
\hline Employed & 35 & 13.2 \\
\hline Unemployed & 231 & 86.8 \\
\hline
\end{tabular}

Currently having a partner

\begin{tabular}{|c|c|c|}
\hline No & 54 & 20.3 \\
\hline Yes & 212 & 79.7 \\
\hline \multicolumn{3}{|c|}{ Sex of the current partner $(n=212)$} \\
\hline Woman & 32 & 12.3 \\
\hline Man & 234 & 88.0 \\
\hline
\end{tabular}

Sexual orientation

\begin{tabular}{l|l|l}
\hline Homosexual & 122 & 45.9 \\
\hline Bisexual & 144 & 54.1 \\
\hline
\end{tabular}

\begin{tabular}{l|c|c}
\hline \multicolumn{2}{l|}{ Sexual role of participants } \\
\hline Insertive & 19 & 7.1 \\
\hline Receptive & 120 & 45.2 \\
\hline Both & 127 & 47.7 \\
\hline
\end{tabular}

Current marital status

\begin{tabular}{l|c|c}
\hline Married & 39 & 14.7 \\
\hline Cohabiting & 88 & 33.1 \\
\hline Single/Separated/Widow & 139 & 52.2 \\
\hline
\end{tabular}

prevention education even though they knew the places (Table 2).

On disclosure of sexual orientation 188/266 (70.7\%) MSM reported having ever disclosed their sexual orientation to health workers whether they were sick or not. Only 58/266 (21.8\%) disclose their sexual orientation when they are sick. Reported barriers to disclosure of their sexual orientation include fear of mistreatment from health workers (122/266 $45.9 \%)$, lack of the confidentially (51/266 - 19.2\%) and stigma and discrimination (93/266 - 34.9\%) (Table 3). 
Table 2. Access to human immunodeficiency virus (HIV) and sexually transmitted infections (STIs) services

\begin{tabular}{|c|c|c|}
\hline & $N=266$ & $\%$ \\
\hline \multicolumn{3}{|l|}{ Access to STI services } \\
\hline $\begin{array}{l}\text { Seek treatment from health } \\
\text { workers when condition worsens }\end{array}$ & 182 & 68.4 \\
\hline Self medicated when sick & 38 & 14.3 \\
\hline Do not seek any STI services & 46 & 17.3 \\
\hline \multicolumn{3}{|c|}{ Access to HIV voluntary counseling and testing } \\
\hline Yes & 81 & 30.5 \\
\hline No & 185 & 69.5 \\
\hline
\end{tabular}

Table 3. Disclosure of men who have sex with men (MSM) sexual orientation to health workers

\begin{tabular}{l|c|c}
\hline & $N=266$ & $\%$ \\
\hline Disclosure of their sexual orientation to health workers \\
\hline Ever disclose & 188 & 70.7 \\
\hline Disclose when sick & 58 & 21.8 \\
\hline Plan to disclose & 20 & 7.5 \\
\hline
\end{tabular}

Perception of the health workers when MSM disclose their sexual orientation

\begin{tabular}{l|c|c}
\hline $\begin{array}{l}\text { Mistreatment from the health } \\
\text { worker }\end{array}$ & 122 & 45.9 \\
\hline Lack of confidentiality & 51 & 19.2 \\
\hline Stigma and discrimination & 93 & 34.9 \\
\hline
\end{tabular}

\section{Discussion}

The aim of this study was to determine the situation regarding MSM access to friendly HIV and STI services from the health facilities in Tanga region, Northern Tanzania. The key results from the study were the low proportion of the MSM who accessed the HIV and STI services, which contributed to negative or lack of user friendly services caused by the negative attitude of health workers towards MSM.

More than half of the MSM recruited in the study did not seek the HIV and STI services provided in the health facilities until they are seriously sick. Alternatively, MSM sought health care services such as the STI treatments, condoms, lubricants privately in authorized facilities and the pharmaceutical market.

This is similar to the study conducted in the USA and Tanzania which explained the delayed treatments for diseases such as HIV and STIs among MSM caused by scared doctors, not ready and feeling ashamed for being MSM $[15,17]$.

Also the survey on stigma, health care access, and HIV knowledge among men who have sex with men conducted in three countries - Malawi, Namibia, and Botswana - observed strong associations between MSM health seeking behavior and stigma and discrimination from the health workers, which is similar to the results of this study [14].

A similar study conducted in South Africa that looked at health service utilization by men who have sex with men in South African cities reported that experienced stigma, discrimination, and negative health worker attitudes are the major barriers for them to access the HIV health services [16].

For the aim of access to health services and living comfortably the health workers should be empowered on MSM sexual orientation in order to deliver the health services safely. Moreover, the finding from the study showed that few MSM plan to disclose their sexual orientation to health workers and about two thirds of them ever disclose their sexual health orientation to health workers due to negative perception they will get from them if the health worker knows he is dealing with MSM. This includes stigma and discrimination, mistreatments and lack of confidentiality. The MSM were ready to disclose their sexual orientation to the nearest relatives rather than health workers.

Similar findings were observed in a study conducted in Dakar, Senegal on barriers to disclosure among MSM that showed that disclosure of sexual orientation among MSM is among the barriers for accessibility of the HIV services to MSM [13].

Furthermore, another study conducted in Dar es Salaam on the barriers of the accessibility of healthcare to MSM showed that the proportion of disclosure of MSM to health workers is low compared to near relatives due to existing fear of the stigma and discrimination and mistreatments from the health workers [18].

Strategies for reaching more MSM and empowering them to overcome perceived fears of the MSM with regards health workers should be in place.

\section{Conclusions}

The study findings showed a low proportion of the MSM who accessed the HIV and STI health services from the health workers due to fear of the stigma and discrimination, lack of confidentiality and mistreatments from the health workers. In order to achieve the goal of ending the HIV epidemic by 2030 the strategies for accessibility of the HIV and STI services to MSM, in whom prevalence is higher than the general population, should be improved. The MSM should be empowered on the accessibility of the HIV and STIs health services. This should go hand in hand with breaking the cycle of misinformation and negative perception about MSM for health workers.

\section{Acknowledgements}

The authors thank TAWG for allowing use of their offices and MSM clients to conduct this study, and secondly the participants for agreeing to participate in the study. We 
also thank District Medical Officers for permission to conduct the study in their respective district and research assistance during the data collection.

\section{Conflict of interest}

The authors declare no potential conflicts of interest with respect to the research, authorship, and/or publication of this article.

\section{References}

1. WHO report. Prevention and Treatment of HIV and other Sexually Transmitted Infections among Men who have Sex with Men and Transgender People. Recommendations for a public health approach. World Health Organization, Geneva 2011.

2. UNAIDS report. The Joint United Nations Programme on HIV/ AIDS, Policy brief: HIV and Sex between Men. August 2006. http:// data.unaids.org/pub.

3. UNAIDS report, Fast-Tracking Combination Prevention: Towards Reducing New HIV Infections to Fewer Than 500000 by 2020 . Geneva, Joint United Nations Programme on HIV/AIDS, 2015.

4. United Nations Population Fund, Global Forum on MSM \& HIV, United Nations Development Programme, World Health Organization, United States Agency for International Development, World Bank. Implementing comprehensive HIV and STI programmes with men who have sex with men: practical guidance for collaborative interventions. New York (NY): United Nations Population Fund; 2015.

5. Beyrer C, Baral SD, van Griensven F, et al. Global epidemiology of HIV infection in men who have sex with men. Lancet 2012; 380: 367-377.

6. Sanders EJ, Graham SM, Okuku HS, et al. HIV-I Infection in high risk men who have sex with men in Mombasa Kenya. AIDS 2007; 21: 2513-2252.

7. Sanders EJ, Okuku H, Smith AD, et al. High HIV-1 incidence, correlates of HIV-1 acquisition, and high viral loads following seroconversion among men who have sex with men in Coastal Kenya. AIDS 2013; 27: 437-446.

8. Beyrer C, Wirtz AL, Walker D, et al. The Global HIV Epidemics among Men Who Have Sex with Men. The World Bank, Washington DC, 2011.

9. Leshabari MT, Mmbaga E, Mpembeni R, Moen K. Prevalence of the Human Immunodeficiency Virus, Other Sexually Transmitted Infections, and Health-related Perceptions, Reflections, Experiences and Practices Among Men Having Sex with Men in Dar es Salaam. Muhimbili University of Health and Allied Sciences (MUHAS) and University of Oslo, Oslo Norway, 2010.

10. TACAIDS. Tanzania Third National Multi-sectoral strategic framework for HIV and AIDS (2013/14 - 2017/18). Prime Minister's Office, United Republic of Tanzania, November 2013.

11. Ross MW, Nyoni J, Ahaneku HO, et al. High HIV seroprevalence, rectal STIs and risky sexual behavior in men who have sex with men in Dar es Salaam and Tanga, Tanzania. BMJ Open 2014; 4: e006175.

12. Lane T, Mogale T, Struthers H, et al. "They see you as a different thing": the experiences of men who have sex with men with healthcare workers in South African township communities. Sex Transm Infect 2008; 84: 430-433.

13. Dahoma M, Johnston LG, Holman A, et al. HIV and related risk behavior among men who have sex with men in Zanzibar, Tanzania: results of a behavioral surveillance survey. AIDS Behav 2011; 15: 186-192.

14. Fay H, Baral SD, Trapence G, et al. Stigma, health care access, and HIV knowledge among men who have sex with men in Malawi, Namibia and Botswana. AIDS Behav 2011; 15: 1088-1097.
15. Nyoni J, Ross MW. Factors associated with HIV testing in men who have sex with men in Dar es Salaam, Tanzania. Sex Transm Infect 2012; 88: 483 .

16. Rispel LC, Metcalf CA, Cloete A, et al. You become afraid to tell them that you are gay: Health service utilization by men who have sex with men in South African cities. J Public Health Policy 2011; 32: S137-S151.

17. Beckerman A, Fontana L. Medical treatment for men who have sex with men and living with HIV/AIDS. Am J Mens Health 2009; 3: 319-329.

18. Magesa DJ, Leshabari M. Barrier to access available health services among the men who have sex with men in Dar es Salaam, Tanzania. Tanzan J Health Res 2017; 19. 